\title{
A rare case of carcinoma cuniculatum of the penis in a 55 -year-old
}

\author{
Paul Lau, Hector H. Li Chang, MD; ${ }^{*}+$ Jose A Gomez, MD, FRCPC; ${ }^{* *}$ Petar Erdeljan, MD; ${ }^{* \neq}$ \\ John R. Srigley, MD, FRCPC,; Jonathan I. Izawa, MD, FRCSC ${ }^{*{ }_{ \pm}}$
}

\begin{abstract}
Carcinoma cuniculatum of the penis is an extremely rare variant of squamous cell carcinoma characterized by an endophytic deeply branching and burrowing growth pattern. One documented case series demonstrated afflicted patients ranging in age from 73-83 years with the tumour located on the glans penis, coronal sulcus or foreskin. We report a case of a 55-year-old with disease located on the ventral aspect of the shaft of the penis. The tumour was invasive into the deep dermal connective tissue, comparatively superficial to all previous documented cases. He subsequently underwent a partial penectomy. The case is discussed with a brief review of the literature.
\end{abstract}

Can Urol Assoc J 2010;4(5):E129-132

\section{Introduction}

In industrialized nations, penile carcinoma is rare, accounting for less than $1 \%$ of all tumours in men. ${ }^{1}$ Ninety-five percent of penile carcinomas are classified as primary epithelial squamous cell carcinoma (SCC). ${ }^{2}$ Variants include basaloid, condylomatous, verrucous, papillary not otherwise specified (NOS), sarcomatoid, adenosquamous and mixed carcinomas. ${ }^{3}$ The verruciform carcinomas of the penis are a heterogeneous group of exophytic, generally low-grade SCC. The group accounts for $25 \%$ to $30 \%$ of penile tumours. They all grossly exhibit a verruciform (wart-like) appearance. Microscopically, they display low- to intermediategrade papillary features. They include condyloma acuminatum, giant condyloma acuminatum, warty (condylomatous) SCC, verrucous carcinoma and low-grade papillary SCC NOS. Historically, subclassifying and differentiating these lesions have been difficult and controversial. ${ }^{4-6}$ The principal features used to differentiate these lesions include the quality of the papillae, the prominence of fibrovascular cores, koilocytic atypia, the interface between tumour and stroma, the grade of atypia and the potential for metastasis. ${ }^{4}$

Recently, Barreto and colleagues have described a distinct variant of penile verruciform tumours that show an endophytic, deeply burrowing, branching phenotype previously described as carcinoma cuniculatum. ${ }^{7}$ In the penis, the lesion is exceedingly rare. Only 1 report of 7 cases is present in the literature. ${ }^{7}$ Carcinoma cuniculatum has been described in many sites of the body, including the sole of the foot (where it was originally described), ${ }^{8}$ oral cavity and pharynx,$^{9,10}$ esophagus, ${ }^{11}$ and other areas of skin, ${ }^{12-15}$, although the definition has not always been uniform in each of these reports. Grossly, carcinoma cuniculatum is indistinguishable from other verruciform carcinomas. Microscopically, characteristic features include a low-grade tumour with a sinus-like endophytic burrowing growth pattern and penetration deep into the lamina propria. No metastatic potential has been documented for carcinoma cuniculatum of the penis. The lesion begins as a condylomatous lesion which eventually ulcerates and develops sinuses that exhibit foulsmelling keratinous material. In the 7 cases of carcinoma cuniculatum of the penis previously reported, all lesions were in men in their seventh or eighth decade of life. In conjunction, lesions were only noted on the glans penis, coronal sulcus or foreskin. We present a case of carcinoma cuniculatum of the penis in a quintagenarian man located at the shaft of the penis.

\section{Case report}

A previously healthy 55 -year-old male was referred to our centre with a $4 \times 2 \mathrm{~cm}$ ulcerated and fungating mass located on the ventral aspect of the shaft of the penis. The mass began as a nodular lesion, which the patient had noticed about a year earlier. The patient observed the mass had doubled in size during a 3-week period prior to the referral. The patient reported mild pruritus, but had not experienced any significant pain or lower urinary tract symptoms. Chest, abdominal, and pelvic computed tomography scans did not demonstrate any lymphadenopathy or distant metastases. There was also no lymphadenopathy when examining the cervical and inguinal regions. The abdominal exam was also unremarkable. On genitourinary exam, we noted an uncircumcised phallus and a ventral mass exhibiting a white discharge. The mass had the appearance of a verrucous car- 


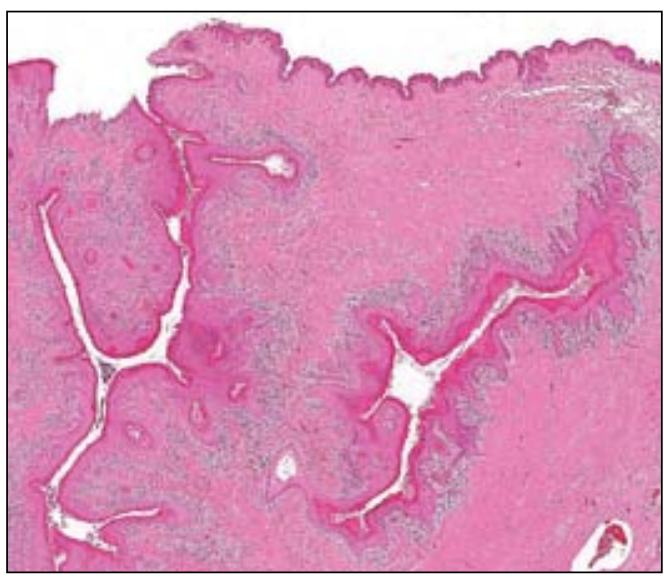

Fig. 1. Low power views of the lesion showing a prominent exophytic and endophytic components. Complex branching, burrowing sinuses invade deeply into the dermis, resembling rabbit burrows. There are both jagged and pushing borders present in the lesion.

cinoma that tunnelled through the penile skin in 3 separate areas. As the lesions were quite mobile, we did not suspect involvement of the urethra or corporal bodies. The patient underwent a partial penectomy using penile and superior scrotal skin to cover the deficit.

Histopathologically, the lesion was nodular and verruciform with an invasive endophytic growth pattern. The tumour had marked hyperkeratosis and architecturally consisted of squamous invaginations and some broad papillae with extension into the deep dermis (Fig. 1) (Fig. 2). A prominent burrowing sinusoidal pattern was present (Fig. 3). In most areas, the tumour had pushing borders; however, other areas had jagged epithelial-stromal interfaces surrounded by lymphoplasmacytic inflammatory infiltrates (Fig. 4). Cytologic atypia was minimal, with rare basally located mitotic activity (Fig. 5) (Fig. 6). Due to the welldifferentiated morphology, prominent keratinisation and unique endophytic growth pattern, the diagnosis of carcinoma cuniculatum of the penis was rendered.

\section{Discussion}

We present the only quintagenarian with carcinoma cuniculatum at the shaft of the penis in the literature. Although all penile carcinoma cuniculatum cases previously documented have been invasive into at least the corpus spongiosum layer, ${ }^{7}$ the tumour in this patient had only invaded into the deep dermal connective tissue. Previously reported cases ranged from 12 to 60 months and disease duration was a significant factor relating to depth of invasion. Also, age-related immune function may potentially play a role in delaying tumour advancement. The relatively young age and shorter disease duration of our patient may explain the comparatively superficial invasion of the primary tumour in this case.

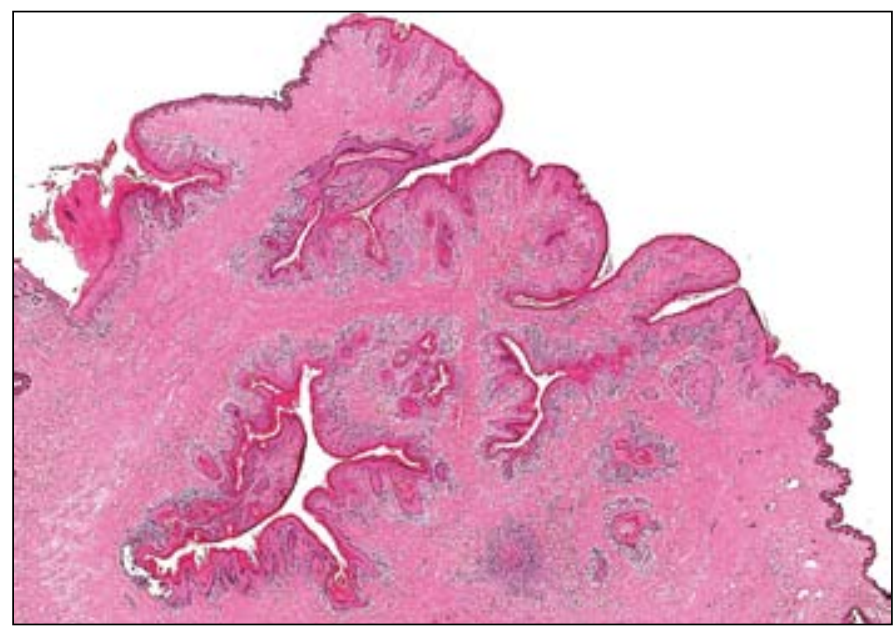

Fig. 2. Low power views of the lesion showing a prominent exophytic and endophytic components. Complex branching, burrowing sinuses invade deeply into the dermis, resembling rabbit burrows. There are both jagged and pushing borders present in the lesion.

Grossly, carcinoma cuniculatum is bulky and white/ gray with a granular, multilobated surface. The lesion has both exophytic and endophytic components, and clinically it may be confused with a verrucous carcinoma or other verruciform lesions. ${ }^{7}$ Multiple sinuses typically form on the surface of the lesion, and they may produce a foul-smelling keratinous discharge. On cut surface, these sinuses display a branching and deeply burrowing pattern that is characteristic of the lesion. These deep invaginations resemble rabbit burrows, from which the term cuniculatum is derived. Microscopically, the surface of the lesion displays hyper-

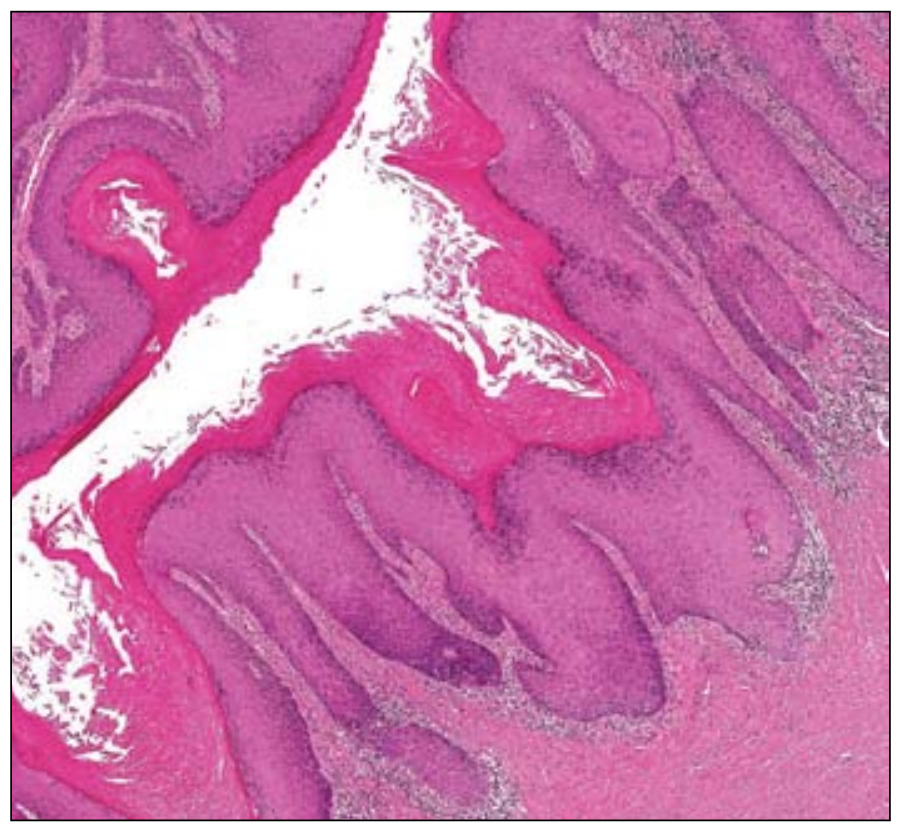

Fig. 3. Mid power view of one of the sinuses. There is prominent orthokeratotic hyperkeratosis. The squamous epithelium is well differentiated with a prominent granular layer. The jagged border is well-appreciated. 


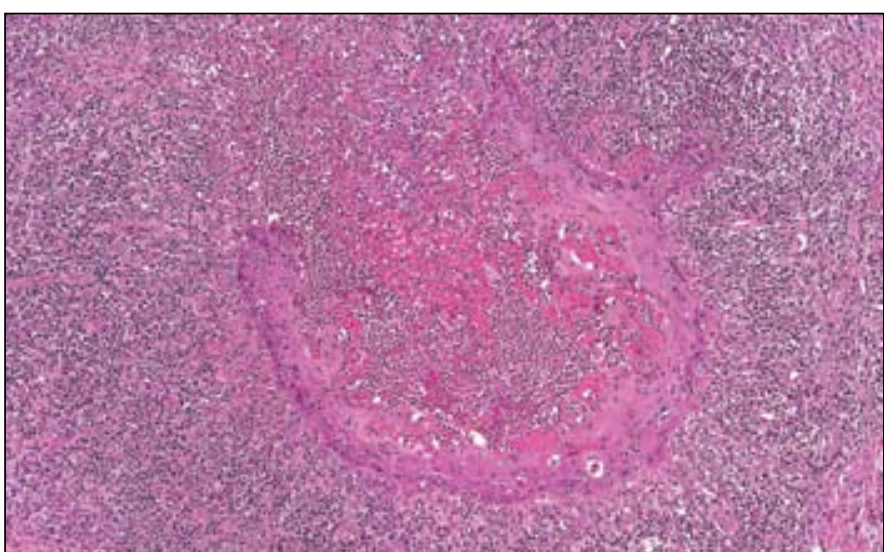

Fig. 4. Invasive focus at the periphery of the lesion composed of well differentiated squamous epithelium with a prominent surrounding inflammatory infiltrate composed primarily of lymphocytes and plasma cells.

keratosis and orthokeratosis. The deeply burrowing sinuses have a hyperkeratotic, a very well-differentiated epithelium. In contrast to superficial invaginations in other tumours, the burrowing sinuses extend deeply beyond the lamina propria. The sinus tracts are also filled with layers of keratin. The borders of the lesion may be jagged or pushing, and are accompanied by an inflammatory reaction composed of lymphocytes and mast cells. Atypia is minimal and mitotic figures are rare. Koilocytic changes are absent. The importance of human papillomavirus in carcinoma cuniculatum in the literature is equivocal. ${ }^{16,17}$

The differential diagnoses for carcinoma cuniculatum of the penis include classic verrucous carcinoma, mixed verrucous carcinoma and condylomatous carcinoma. The nomenclature and morphologic definition of carcinoma cuniculatum is not uniform, and some authors have even equated it with verrucous carcinoma. However, it should be distinguished as a distinct entity for both therapeutic and academic reasons. Carcinoma cuniculatum displays hybrid features of SCC NOS and verrucous carcinoma. In contrast to verrucous carcinoma, carcinoma cuniculatum has at least focally jagged borders and has a propensity to invade more deeply and involve the corporal bodies. In contrast to SCC NOS, carcinoma cuniculatum of the penis has not been associated with lymphatic or distant metastases despite deep invasion in the penis, though it has been reported to rarely have metastasized from the skin elsewhere. ${ }^{15,18}$ The most important differentiating feature, however, remains the characteristic deep burrowing pattern displayed by the tumour.

The correct diagnosis of a carcinoma cuniculatum lesion of the penis is important for post-resection management. Although locally destructive, the absence of metastatic potential in the penis means that surgical resections can be performed with curative expectations. This also applies to classic verrucous tumours. ${ }^{3}$ In contrast, papillary SCC NOS, mixed verrucous carcinomas and condylomatous

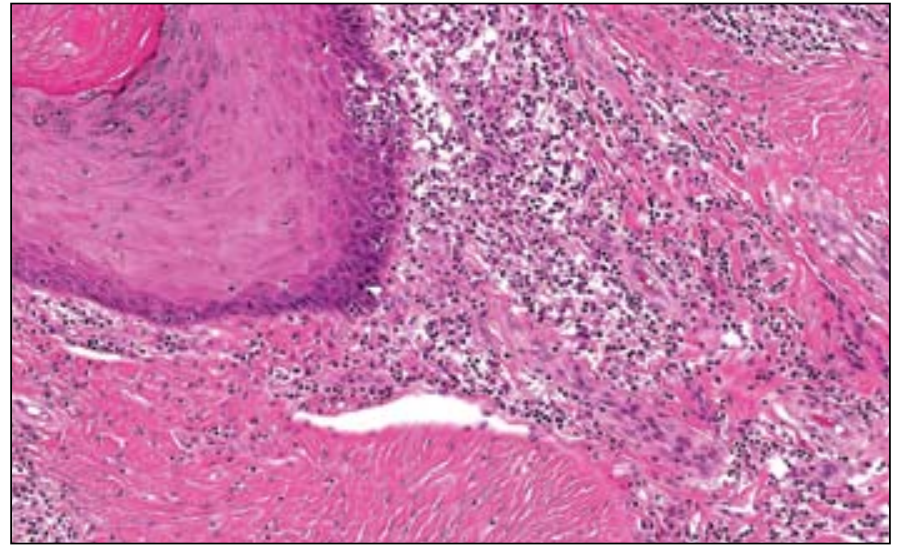

Fig. 5. High power views of the sinuses. The squamous epithelium is very well differentiated with orthokeratotic hyperkeratosis. Scattered mitotic figures are present in the basal layer. There is a prominent surrounding inflammatory infiltrate composed primarily of lymphocytes and plasma cells.

carcinomas of the penis have the potential to metastasize to regional lymph nodes. ${ }^{19,4}$ These 2 tumour types can also occur as intermediate- to high-grade lesions. ${ }^{3,4}$ The association of histological grade with respect to risk of metastasis has been well-documented in penile carcinoma cases. ${ }^{20}$ In patients undergoing partial or radical penectomy, pathologies revealing intermediate- to high-grade mixed verrucous carcinoma or condylomatous carcinoma may require ilioinguinal lymph node dissection (ILND), a procedure associated with significant morbidity. ${ }^{21}$ Due to this morbidity, surveillance strategies seem to be most appropriate for low-grade, non-invasive primary tumour, where the risk of regional lymph node metastases is very low. ${ }^{22}$ Due to what appears to be minimal to no metastatic potential for penile carcinoma cuniculatum, active surveillance seems to be more appropriate than early ILND.

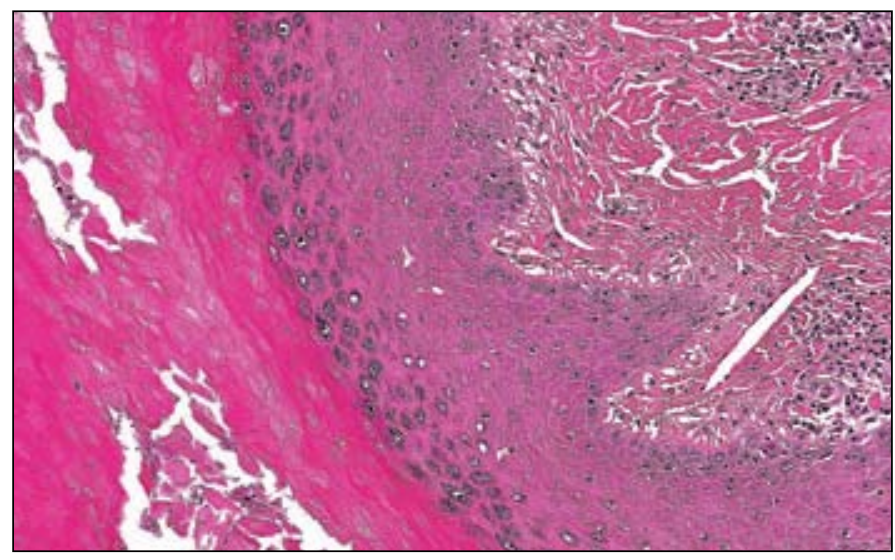

Fig. 6. High power views of the sinuses. The squamous epithelium is very well differentiated with orthokeratotic hyperkeratosis. Scattered mitotic figures are present in the basal layer. There is a prominent surrounding inflammatory infiltrate composed primarily of lymphocytes and plasma cells. 
Lau et al.

\section{Conclusion}

Carcinoma cuniculatum of the penis is an extremely rare variant of SCC. The main defining feature of this tumour is the deeply burrowing growth pattern observed microscopically. Differentiating carcinoma cuniculatum from other grossly similar penile tumours is important for determining surveillance versus early ILND. Examining outcomes of future cases as well as previously misdiagnosed cases of carcinoma cuniculatum of the penis may allow for definitive assessment of this tumour's metastatic potential.

"Schulich School of Medicine and Dentistry, University of Western Ontario, London, ON; 'Department of Pathology, University of Western Ontario, London, ON; : Division of Urology, University of Western Ontario, London, ON Departments of Surgery \& Oncology, University of Western Ontario, London Health Sciences Centre-Victoria Hospital and the London Regional Cancer Program, London, ON; ¿Department of Laboratory Medicine, Credit Valley Hospital, Mississauga, ON

Competing interests: None declared.

This paper has been peer-reviewed.

\section{References}

1. Persky L. Epidemiology of cancer of the penis. Recent Results Cancer Res 1977;60:97-109.

2. Burgers JK, Badalament RA, Drago JR. Penile cancer. Clinical presentation diagnosis and staging. Urol Clin North Am 1992;19:247-56.

3. Eble JN, Sauter G, Epstein Jl, et al. Pathology and Genetics of Tumours of the Urinary System and Male Genital Organs. Lyon, IARC Press; 2004:285-7.

4. Cubilla AL, Velazquez EF, Reuter $V$, et al. Warty (condylomatous) squamous cell carcinoma of the penis. Am J Surg Pathol 2000;24:505-12.

5. Steffen C. The men behind the eponym-Abraham Buschke and Ludwig Lowenstein: giant condyloma (Buschke-Loewenstein). Am J Dermatopathol 2006;28:526-36.
6. Steffen C. Dermatopathology in historical perspective: epithelioma cuniculatum (Aird). Am J Dermatopathol 2006;28:451-61.

7. Barreto JE, Velazquez EF, Ayala E, et al. Carcinoma cuniculatum: A distinctive variant of penile squamous cell carcinoma. Am J Surg Pathol 2007;31:71-5.

8. Aird I, Johnson HD, Lennox B, et al. Epithelioma cuniculatum: a variety of squamous carcinoma peculiar to the foot. Br I Surg 1954;42:245-50.

9. Gassler N, Helmke B, Schweigert HG, et al. Carcinoma cuniculatum of the oral cavity. A contribution to the differential diagnosis of potentially malignant papillary lesions of the mouth mucosa. Pathologe 2002:23:313-7.

10. Kuffer R, Brocheriou C, Rougier M, et al. Buccopharyngeal localizations of carcinoma cuniculatum. Apropos of 5 cases. Arch Anat Cytol Pathol 1984;32:184.

11. De Petris G, Lewin M, Shoii T. Carcinoma cuniculatum of the esophagus. Ann Diagn Pathol 2005;9:134-8.

12. Lawrence-Brown MM, Gollow IJ, Lat T, et al. Carcinoma cuniculatum of the abdominal wall. Med I Aust 1984;140:668-9.

13. Melo CR, Melo IS. Carcinoma cuniculatum of the buttock. An unusual case with an inverted growth. Dermatologica 1989;179:38-41.

14. Melo CR, Melo IS. Carcinoma cuniculatum originating from intertriginous lesions. Int I Dermatol 1991;30:707-9.

15. Kao GF, Graham JH, Helwig EB. Carcinoma cuniculatum (verrucous carcinoma of the skin): a clinicopathologic study of 46 cases with ultrastructural observations. Cancer 1982:49:2395-403.

16. Knobler RM, Schneider S, Neumann RA, et al. DNA dot-blot hybridization implicates human papillomavirus type 11-DNA in epithelioma cuniculatum. J Med Virol 1989;29:33-7.

17. Petersen CS, Sjolin KE, Rosman N, et al. Lack of human papillomavirus DNA in carcinoma cuniculatum. Acta Derm Venereol 1994;74:231-2.

18. McKee PH, Wilkinson JD, Corbett MF, et al. Carcinoma cuniculatum: a cast metastasizing to skin and lymph nodes. Clin Exp Dermatol 1981;6:613-8.

19. Clemente Ramos LM, Garcia Gonzalez R, Burgos Revilla FJ, et al. Hybrid tumour of the penis: is this denomination correct? Arch Esp Urol 1998:51:821-3.

20. Novara $G$, Galfano A, De Marco $V$, et al. Prognostic factors in squamous cell carcinoma of the penis. Nat Clin Pract Urol 2007;4:140-6.

21. Bevan-Thomas R, Slaton JW, Pettaway CA. Contemporary morbidity from lymphadenectomy for penile squamous cell carcinoma: the MD Anderson Cancer Center experience. J Urol 2002;167:1638-42.

22. Leitje JA, Kirrander $\mathrm{P}$, Antonini $\mathrm{N}$, et al. Recurrence patterns of squamous cell carcinoma of the penis: recommendations for follow-up based on a two-centre analysis of 700 Patients. Eur Urol 2008;54:161-9.

Correspondence: Dr. Jonathan I. Izawa, Associate Professor, Departments of Surgery \& Oncology, Divisions of Urology \& Surgical Oncology, The London Health Sciences Centre, 800 Commissioners Road East, Room E2-649, London, 0N N6A 465; fax: 519-685-8455; jonathan.izawa@ @hsc.on.ca 